\title{
Colonic actinomycosis: a pseudo-tumour that mimics colonic neoplasm
}

KW Hui *, MB, ChB, Bryant SY Chan, MB, BS, FRCS, Kenny KY Yuen, MB, BS, FRCS

Department of Surgery, Tseung Kwan O Hospital, Tseung Kwan O, Hong Kong

*Corresponding author: karen.hui@icloud.com

Hong Kong Med J 2019;25:334-6

https://doi.org/10.12809/hkmj187523

\section{Introduction}

Actinomyces is a class of Gram-positive, non-acid-fast, anaerobic-to-microaerophilic bacteria commonly found in the oral cavity. It is known for its causative role in oral infections after dental procedures. Abdominopelvic involvement is uncommon, with few reported cases. Risk factors of intra-abdominal actinomycosis include use of intrauterine device, abdominal or pelvic procedures, and various causes of intestinal necrosis. ${ }^{1}$ Current understanding on intra-abdominal actinomycosis has highlighted its inclination to mimic neoplasm. Reported cases on organ dissemination and progression to fistulae formation further confound this diagnostic challenge in clinical practice. Although perforation of neoplasm remains the primary concern in septic manifestations of an abdominal mass lesion, infective differentials such as actinomycosis should be considered. Because the presentation of acute abdomen necessitates timely treatment, early operative treatment is often favoured by clinicians, particularly when complications such as sepsis and intestinal obstruction arise. This leaves limited scope for investigations. As a result, the diagnosis of intraabdominal actinomycosis is often established from pathological assessment after cure.

\section{Exemplar local case}

A 35-year-old woman with good past health presented to Tseung Kwan O Hospital, Hong Kong, in December 2017, with 1-month history of right lower abdominal discomfort and low-grade fever. Abdominal examination identified an 8-cm tender right lower quadrant mass with local guarding and rebound tenderness. Blood tests showed neutrophil predominant leukocytosis $\left(17.2 \times 10^{9} / \mathrm{L}\right)$. Urgent contrast enhanced computed tomography scan of the pelvis revealed an ill-defined heterogeneously enhancing mass at right lower abdomen with matted manifestations in its vicinity, involving an oedematous terminal ileal segment and gynaecological organs (Fig 1). The patient underwent diagnostic laparoscopy with revelation of an $8-\mathrm{cm}$ locally invasive firm mass arising from the caecum. Dense adhesions to the colonic mesentery and terminal ileum were dissected free. The right infundibulopelvic ligament and right base of ovary appeared to be invaded by the colonic pseudo-tumour. The procedure proceeded to an en bloc resection including formal right hemicolectomy and right salpingo-oophorectomy. The patient had an uneventful postoperative recovery course. Pathological examination of the specimen (Fig 2) unveiled histological diagnosis

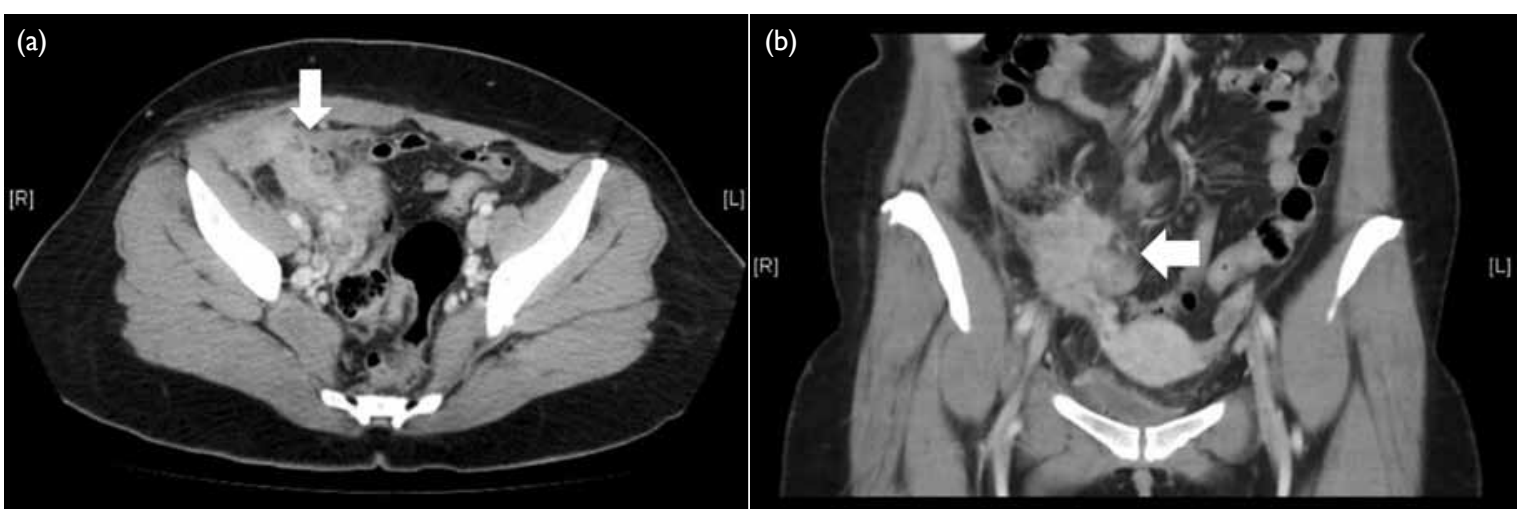

FIG I. (a) Axial and (b) coronal views of contrast computed tomography images showing ill-defined heterogeneously enhancing matted lesion at right lower quadrant 


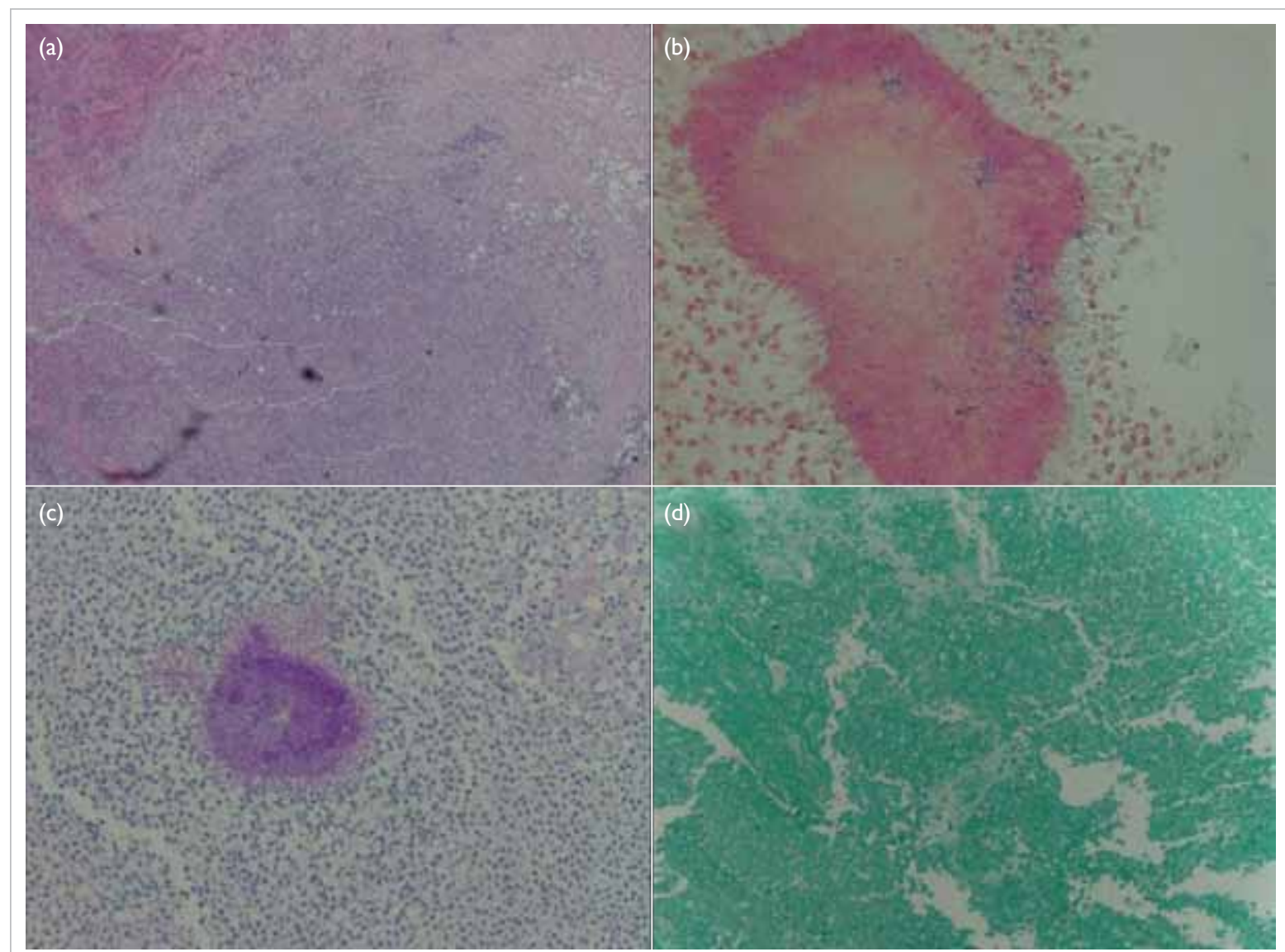

FIG 2. (a) Section of the thickened caecal wall showing areas of abscess formation surrounded by fibrosis and chronic inflammation. Bacterial colonies are focally found within the neutrophilic infiltrate $(\times 40)$. (b) Gram-positive filamentous bacteria in the colonies, consistent with actinomycosis (Gram stain, $\times 400)$. (c) Periodic acid-Schiff stain $(\times 400)$ and (d) Grocott stains $(\times 200)$ are both negative for fungal organisms

of disseminated actinomycosis with abscess, fibrosis, and inflammation extending into adjacent mesenteric tissue, mesoappendix, mesosalpinx, and adhering fibrofatty tissue.

\section{How to investigate actinomycosis}

The current role of imaging in the diagnosis of intraabdominal or pelvic actinomycosis is poorly defined. Limited studies have suggested some computed tomographic features of actinomycosis describing its infiltrative nature and tendency to invade across tissue plane and boundaries. Dense inhomogeneous contrast enhancement in walls or solid component of masses and minimal lymphadenopathy may also be characteristic. ${ }^{2}$ However, the features are often insufficient to help distinguish the condition from most other differentials. Kim et $\mathrm{al}^{3}$ investigated the efficacy of combining colonoscopy and computed tomography. In future, combined investigations are expected to gain popularity, to take advantage of complementary imaging modalities. Degree of stenosis and mucosal abnormalities are other areas being further evaluated for imaging studies. Cytological investigations are effective but limited to variants of actinomycosis such as multi-cystic subtypes. Image-guided fine needle aspiration may identify actinomycotic granules on microscopy and facilitate bacterial culture. ${ }^{4}$ Currently, most clinicians remain reluctant to consider cytological or tissue samples to avoid the possibility of tumour seeding before a certain diagnosis has been made. ${ }^{5}$

\section{How to treat actinomycosis}

Owing to the scarcity of reported cases of actinomycosis, a standardised management protocol is lacking to date. Evans et $\mathrm{al}^{5}$ advocate antibiotic therapy to obliterate the disease, while proceeding to extensive resection for the purpose of debulking. High-dose penicillin is an established first-line regimen in the treatment of actinomycosis. Doxycycline and clindamycin are alternatives, especially in patients with penicillin allergy. 
Prolonged course of antibiotics postoperatively up to 6 to 12 months until sign of disease clearance is advocated by some. In view of the tendency of actinomycosis to spread across tissue planes and disseminate to other organs, surgical resection may be extensive. Preoperative counselling on possible extent of surgery is crucial in all cases where actinomycosis is considered.

\section{Prospects}

Intra-abdominal actinomycosis is uncommon worldwide. Imaging and cytological investigations are shown to be useful but their roles in establishing definitive diagnosis are yet to be defined. Preliminary studies on combined imaging have demonstrated promising results. However, a lack of consensus remains over antibiotic therapy alone versus antibiotic therapy with additional debulking surgery. Prognosis of the disease entity remains unclear. An evidence-based management protocol will help standardise care of this rare disease entity as we gain more understanding from additional cases over time.

\section{Author contributions}

All authors had full access to the data, contributed to the study, approved the final version for publication, and take responsibility for its accuracy and integrity.

Concept or design of the study: KW Hui, BSY Chan. Acquisition of data: KW Hui, BSY Chan.

Analysis or interpretation of data: KW Hui, BSY Chan.
Drafting of the article: KW Hui.

Critical revision for important intellectual content: All authors.

\section{Conflicts of interest}

The authors have no conflicts of interest to disclose.

\section{Funding/support}

This research received no specific grant from any funding agency in the public, commercial, or not-for-profit sectors.

\section{Ethics approval}

This study was conducted in accordance with the principles outlined in the Declaration of Helsinki. Informed consent has been obtained from patient for the treatment.

\section{References}

1. Filippou D, Psimitis I, Zizi D, Rizos S. A rare case of ascending colon actinomycosis mimicking cancer. BMC Gastroenterol 2005;5:1.

2. Ha HK, Lee HJ, Kim H, et al. Abdominal actinomycosis: CT findings in 10 patients. AJR Am J Roentgenol 1993;161:791-4

3. Kim JC, Ahn BY, Kim HC, et al. Efficiency of combined colonoscopy and computed tomography for diagnosis of colonic actinomycosis: a retrospective evaluation of eight consecutive patients. Int J Colorectal Dis 2000;15:236-42.

4. Cameron AE, Menon GG, Wyatt AP. Abdominal actinomycosis mimicking carcinomatosis. J R Soc Med 1988;81:231-2.

5. Evans J, Chan C, Gluch L, Fielding I, Eckstein R. Inflammatory pseudotumour secondary to actinomyces infection. Aust N Z J Surg 1999;69:467-9. 